\title{
LA CONSTRUCCIÓN SOCIAL DEL ESPACIO MINERO: SINTAXIS DE LA ARQUITECTURA DOMÉSTICA EN EL NORTE DE MENDOZA (ARGENTINA)
}

\author{
THE SOCIAL CONSTRUCTION OF MINING SPACE: SYNTAX OF DOMESTIC \\ ARCHITECTURE IN THE NORTH OF MENDOZA (ARGENTINA)
}

\begin{abstract}
Osvaldo Sironi*
El estudio arqueológico sistemático de dos emplazamientos mineros localizados en el centro oeste de Argentina permite ahondar en el conocimiento de las condiciones de vida de sus habitantes. Aquí se aborda un análisis arquitectónico comparado de edificios que funcionaron entre mediados de siglo XIX e inicios del siglo XX para diferentes fines extractivos (oro, plata y esquisto bituminoso) con una población residente constituida por familias o grupos de trabajadores. Por medio de análisis constructivos y sintácticos del espacio, de escasa aplicación en la región, proponemos que la arquitectura en los emplazamientos mineros funcionó como un objeto activo en las estrategias de control de sus habitantes, ya que se configuró un patrón arquitectónico asimilable al de "tipo cuartel”, el que permitía el control de los accesos y caminos del lugar, aumentando el aislamiento y la segmentación de los espacios.

Palabras claves: Sintaxis espacial, Arquitectura, Minería, Mendoza (Argentina).

The systematic archaeological study of two mining sites located in the central west of Argentina allows to know the living conditions of mining peoples. Here we discuss a comparative architectural analysis of buildings that operated between the middle 19th and early 20th centuries, within mining plans with different extractive purposes (silver, gold and schist). They maintained a resident population consisting of families or groups of workers. Through constructive and syntactic analyzes of space, with very little application in the region, we propose that the architecture in the mining sites functioned as an active object and a key input in the control strategies of its inhabitants. These were configured architecturally and spatially according to the economic interests of the owners of the means of production, who had the exploitation rights and therefore designed an architectural pattern "barracks type", since it allowed the control of the accesses and roads of the place, increasing the enclosure, isolation and the segmentation of the spaces.

Key words: Space syntax, Architecture, Mining, Mendoza (Argentina).
\end{abstract}

\section{Introducción}

Con este trabajo pretendemos contribuir a la caracterización de los materiales y las técnicas de construcción aplicados a las edificaciones mineras. Además, a base de los aportes del análisis sintáctico del espacio -forma, acceso, circulación y visibilidad-(Blanton 1994; Ching 1995; Hillier y Hanson 1984), analizaremos la estructuración sociocultural de los ámbitos productivos y domésticos de dichos emplazamientos, identificando las correlaciones arquitectónicas intrasitios con el fin de comprender la organización de los sujetos sociales en los emplazamientos mineros. Este trabajo se enmarca en una perspectiva teórico-metodológica orientada al análisis de las condiciones y modos de vida de poblaciones mineras en un período histórico específico de industrialización capitalista, partiendo del systematic study of structures and artefacts as a means of enlarging our understanding of the industrial past (Palmer y Neaverson 1998:1). En el Noroeste de Mendoza, este período se caracteriza por el desarrollo y transformación de las fuerzas productivas y las relaciones sociales de producción en contextos socioculturales mineros del siglo XVII al siglo XIX inclusive, de los pirquineros a las comunidades mineras industriales (Sironi 2019).

Los resultados obtenidos en los análisis de los registros arqueológicos de las instalaciones mineras precordilleranas (Figura 1) conocidas como Minas Paramillos Sur -en adelante MPS- y Mina La Atala -en adelante MLA- ofrecen, entre otras evidencias, restos de arquitectura que nos permiten conocer procesos de ocupación y modos de organización y delimitación espacial relacionadas con las condiciones de vida de sus habitantes.

\footnotetext{
* Instituto Argentino de Nivología y Glaciología-CONICET, Mendoza, Argentina. Correo electrónico: osvaldosironi@ gmail.com
} 


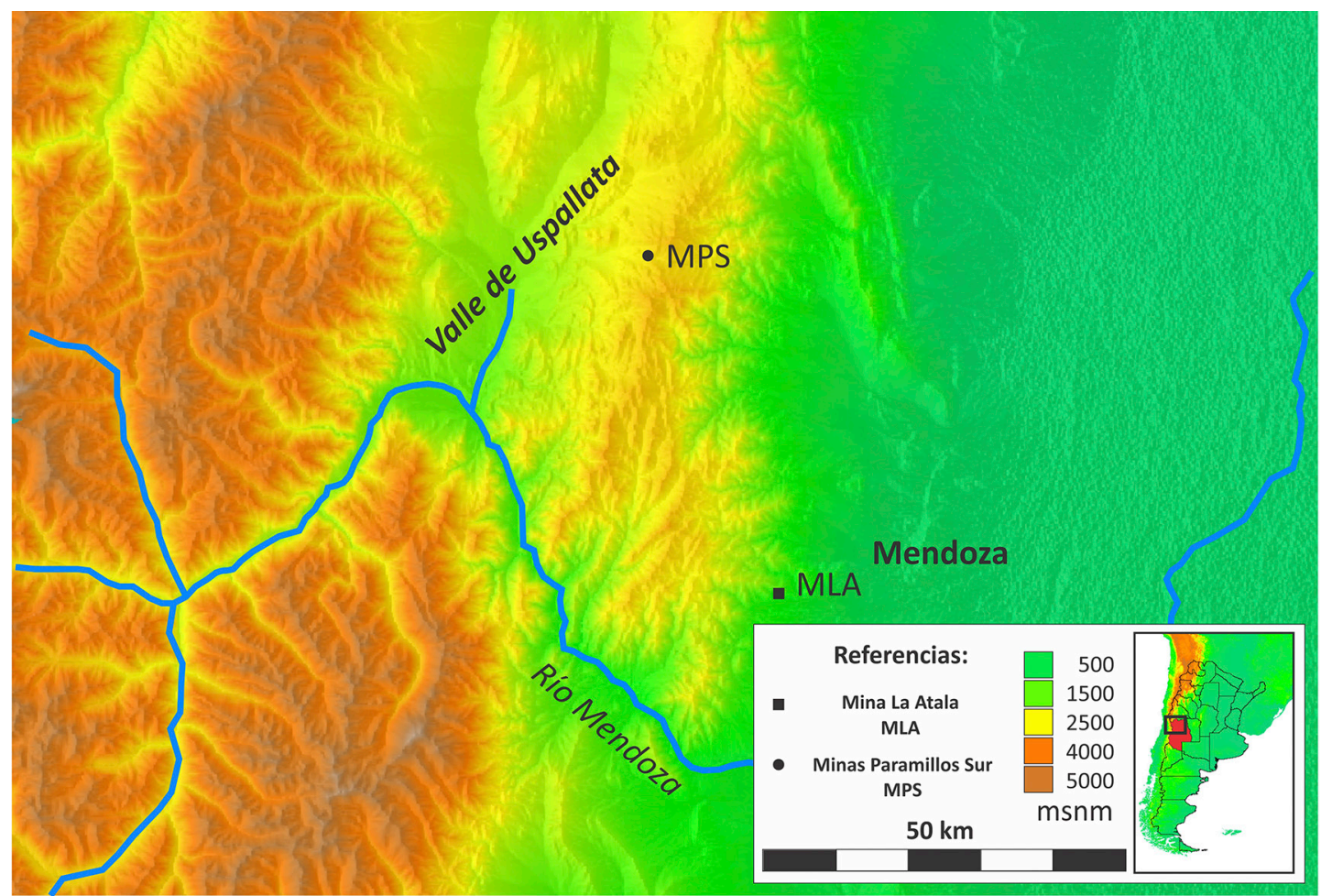

Figura 1. Mapa del Noroeste de Mendoza y el sector de MPS (Minas Paramillos Sur) y MLA (Mina La Atala). Fuente: Osvaldo Sironi y Pablo Cahiza (2018).

\section{Materiales y método}

Para cumplir con los objetivos propuestos, efectuamos el relevamiento descriptivo y el diagnóstico del estado de conservación de la totalidad de los conjuntos arquitectónicos evidenciados en los sitios histórico-arqueológicos MPS y MLA (Figura 2). Por conjunto arquitectónico se entiende a la unión de dos o más recintos delimitados por medianeras o muros. A su vez recinto se refiere a un espacio contiguo sin segmentación interna (Nastri 1997-1998, Wynveldt 2005). El total de conjuntos arquitectónicos asciende a 28 -con sus respectivas subdivisiones-, que están distribuidas entre MPS $(\mathrm{N}=18)$ y MLA $(\mathrm{N}=10)$. Dichos conjuntos arquitectónicos fueron relevados en las primeras temporadas de trabajos arqueológicos realizados en los sitios, obteniendo datos a partir de prospecciones, recolecciones superficiales, pozos de sondeo -en sectores internos y externos de los conjuntos arquitectónicos de MPS $\left(7 \mathrm{~m}^{2}\right)$ y MLA $\left(4 \mathrm{~m}^{2}\right)$-, medidas de los recintos arquitectónicos y las técnicas de construcción utilizadas (Sironi 2015, 2018).

El trabajo en gabinete consistió en la digitalización de los registros arquitectónicos realizados en el campo y la aplicación de los análisis espaciales de circulación -interna y externa- a las estructuras mineras relevadas, específicamente a los conjuntos arquitectónicos considerados como espacios domésticos, debido a la presencia de materialidad arqueológica vinculada a dicho modo de vida -utensilios: MPS (Estructuras III, IV, V y VI) y MLA (Conjunto A -Estructura III-). Los estudios comparativos entre diferentes estructuras arquitectónicas fueron procesados a partir de los métodos propuestos por Hillier y Hanson (1984) y Blanton (1994), con el fin de comprender la estructuración sociocultural del espacio minero del norte de Mendoza.

A partir de estos trabajos se obtuvo información que posibilitó identificar y caracterizar las posibles funciones de las habitaciones que componen los complejos arquitectónicos-estructurales; comprobar las secuencias cronológicas de ocupación para corroborar sincronías o diacronías de rasgos correspondientes a emplazamientos mineros y sugerir explicaciones de los modos de vivir en emplazamientos mineros, a partir de los análisis de la construcción de los espacios domésticos-productivos y de la materialidad arqueológica recolectada de los sitios mencionados (Chiavazza y Prieto Olavarría 2012; Sironi 2019). 

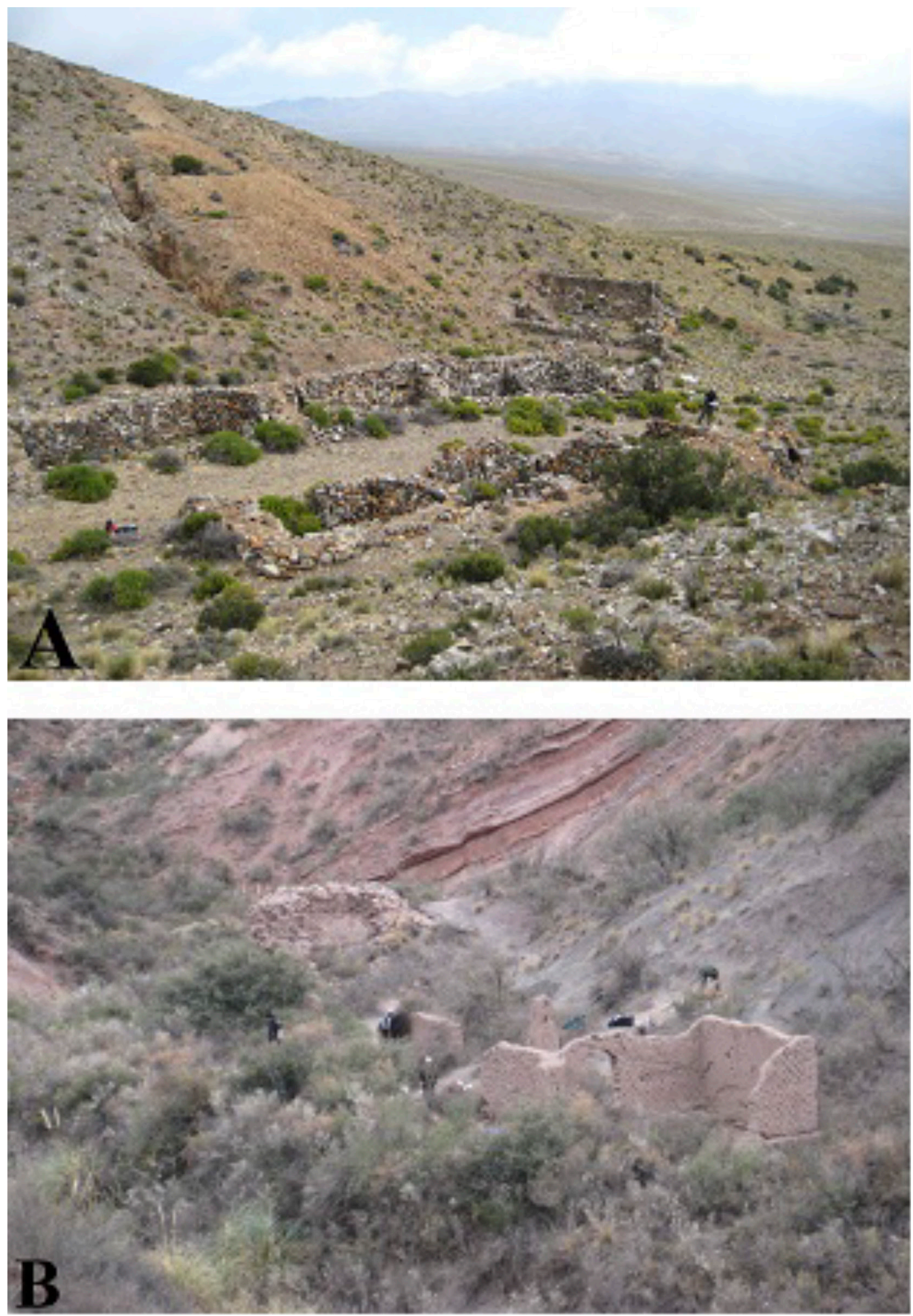

Figura 2. Complejos mineros durante las excavaciones arqueológicas: A. Minas Paramillos Sur (2012). B. Mina La Atala (2013). Fuente: Osvaldo Sironi (2012 y 2013). 
El control del espacio es un elemento central en la dinámica de reproducción del poder. Mediante la manipulación del mundo material se generan las condiciones necesarias para que este proceso tenga lugar de manera exitosa. La arquitectura, como elemento central de esa dimensión material, es entendida como tecnología del poder que actúa como un tipo de comunicación no verbal, manifestando un lugar destacado en la transmisión de discursos ligados a la creación y manutención de relaciones de poder (Foucault 1976, Fletcher 1989). En efecto, los planos verticales -muros- actúan como segregadores de los espacios, y las aperturas en él aplicadas como elementos de restricción y control, condicionando el grado de privacidad que tiene un determinado espacio (Kent 1990, Samson 1990, Sánchez 1998, Ching 2000).

El análisis gamma (Hillier y Hanson 1984) busca entender el movimiento por medio de los espacios, cuantificando las profundidades y permeabilidades. Es un diagrama interpretativo (cualitativo y cuantitativo) que representa espacios y vías de acceso mediante esquemas conformados por "nodos" y "conectores". Los "nodos" son espacios determinados por muros, u otros marcadores de límites. El espacio exterior es representado por un nodo, aunque se contabiliza con el cero. Los "conectores" representan los pasajes entre nodos e incluye aberturas y pasadizos. Esta forma de representación gráfica tiene la ventaja de plasmar en forma sencilla las relaciones entre distintos ámbitos y los pasos que deben dar quienes se mueven en ellos (Hillier y Hanson 1984). Esto da como resultado mapas cuya eficacia "radica en su habilidad de capturar las relaciones sociológicamente relevantes integradas en una planta" de ocupación (Bafna 2003:23).

Los elementos clave en este análisis son los umbrales que separan o comunican los espacios entre sí, pues actúan como controladores de paso a determinado ambiente, basado en el carácter de interioridad/exterioridad de cada lugar y su relación con las áreas adyacentes, es decir, su permeabilidad (Hillier y Hanson 1984). Los tipos de permeabilidad de un espacio son representados a partir de círculos (espacios), líneas (accesos-conexiones) y líneas guionadas (espacios contiguos sin conectividad) dentro de un mapa gamma. Los diagramas de permeabilidad se basan, por tanto, en el ordenamiento espacial, en el control del acceso y en el movimiento que este permite. Con ellos se pueden identificar las relaciones sintácticas del espacio: miden el grado de dependencia de unas áreas respecto de otras y el control que sobre ellas se ejerce (simetría/asimetría) y la cantidad y disposición de los accesos (distribuida/no distribuida). Por medio de estos análisis se pueden interpretar posibles relaciones sociales que mantienen los sujetos que habitan una estructura y, asimismo, plantear tipos de relaciones entre los sujetos ajenos a esta estructura (Criado Boado 1991), ya que el ...space creates special relations between function and social meaning... (Hillier y Hanson 1984:1-2). Posteriormente, el modelo gamma fue ampliado y complementado metodológicamente por Richard Blanton (1994), quien toma como base aquel modelo y genera tres índices que posibilitan afinar y profundizar el análisis de la estructura arquitectónica: "Escala, Integración y Complejidad".

El índice de "escala" permite evidenciar la gran diferencia existente entre estructuras, dando cuenta de la composición y del tamaño del conjunto a partir de la cantidad de estructuras/recintos y sus superficies. El índice de "integración" permite dar cuenta de la relación entre cantidad de conexiones y cantidad de estructura/recinto, evidenciando las relaciones de circulación y restricciones del sistema. El índice de "complejidad" deja ver las diferencias en cuanto a número de conexiones existentes y la variación funcional que tienen los espacios, revelando los grados de control y acceso respecto del exterior. La variable que guía este análisis es el concepto de restricción, control y segregación espacial, que supone que un aumento del control y privacidad se materializa en mayores restricciones espaciales y menor cantidad de conexiones (Blanton 1994, Zarankin 1999, Baxter 2002).

En las graficaciones se representan los diversos espacios (círculos, nodos) de las estructuras asociadas con espacios domésticos de cada asentamiento minero analizado. Se consideran las relaciones de espacialidad por medio de la contigüidad entre las habitaciones que no se encuentran comunicadas entre sí (se usan líneas discontinuas); y aquellos ingresos o conectores entre epacios (las líneas continuas). Con estas variables se obtienen gráficos indicativos de la permeabilidad/conectividad existente entre las estructuras, es decir, comunicación interna.

En nuestro país esta técnica se aplicó a estudios de arquitectura urbana de la Ciudad de Buenos Aires (Zarankin 1999; Funari y Zarankin 2003; Zarankin y Niro 2009), como así también en contextos rurales del Valle de Uco (Chiavazza 2010) ${ }^{1}$. 
En ellos se detecta la potencialidad que este tipo de análisis puede tener para el estudio arquitectónico y espacial, y este caso constituye su primera aplicación en el ámbito de la arquitectura minera de Mendoza. De este modo, la aplicación de estos análisis nos permitirá identificar el hilo perceptivo que se implementa en una construcción minera, es decir, si esta percepción está condicionada por algún elemento significativo (algún elemento arquitectónico diferencial) y su significación. Coincidimos en que estos aspectos contribuyen a entender tópicos de la vida doméstica, tanto por las funciones como por las significaciones que las arquitecturas denotan y connotan (Eco 1968). Esto lo entendemos como de singular relevancia para aportar al conocimiento de las relaciones y condiciones de vida en entornos laborales mineros en el marco de una historia social de la región.

\section{Entornos ambientales y emplazamientos mineros locales}

En el sector históricamente ocupado para la extracción de recursos mineros metalíferos (oro, plata, plomo y cobre), dentro de la microrregión Paramillos de Uspallata, se ubica el sitio históricoarqueológico denominado Minas Paramillos Sur

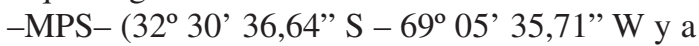
una altitud aproximada de 3.000 metros sobre el nivel del mar) con instalaciones arquitectónicas construidas en su totalidad con rocas locales. Dentro de un ambiente propio de la "Provincia Fitogeográfica Puneña", caracterizado por el clima frío (gran amplitud térmica diaria), seco (entre 100 y $200 \mathrm{~mm}$ de precipitaciones anuales concentradas en época estival), y con diferencias estacionales profundas (Cabrera y Willink 1980). La información documentada que existe de las minas de Los Paramillos se remonta a las explotaciones intermitentes que se llevaron a cabo en el área desde el siglo XVII en adelante, señalando la existencia de períodos de ardua labor como también de total abandono por la falta de capitales para invertir, brazos para trabajar, técnicos idóneos y una red de comunicaciones eficiente (Cueto 2003, Sironi 2013). A partir de mediados del siglo XIX, el área geológica adquiere una mayor relevancia, ya que se inician explotaciones de minerales (plata y oro principalmente) a gran escala a partir de capitales extranjeros (Lallemant 1890, Cueto 2003, Grilli 2006, Ataliva 2011, Sironi 2013,).
En la cuenca aluvional denominada Divisadero Largo, dentro de la Reserva Natural homónima, en un área comprendida entre los $32^{\circ} 52^{\prime} 22^{\prime \prime}$ y $32^{\circ} 52^{\prime} 46^{\prime \prime} \mathrm{S}$ y $\operatorname{los} 68^{\circ} 56^{\prime} 12^{\prime \prime}$ y $68^{\circ} 55^{\prime} 28^{\prime \prime}$ ' W, se enclava el sitio histórico-arqueológico "Mina La Atala" -MLA-. Esta área se encuentra en el ecosistema de Piedemonte, tratándose de una de las regiones de la provincia mejor conocidas desde el punto de vista estratigráfico-tectónico y de sus combustibles sólidos minerales -como es el caso de los esquistos bituminosos- (Chiotti 1946). Las instalaciones de MLA están ubicadas en el margen norte del Arroyo a 1.000 msnm aproximadamente y construidas en adobe, piedra, ladrillos y hormigón. Las mismas están asociadas a dos filones de pizarras bituminosas conocidos como Jirón A y Jirón B y a cuatro canteras de materiales arcillosos. La mina La Atala presenta un espesor aproximado de $50 \mathrm{~m}$ de lutitas bituminosas, que, en un primer momento, fueron explotadas para producir el gas del alumbrado público de la ciudad de Mendoza por la "Compañía de Gas de Mendoza" -empresa fundada el 1 de marzo de 1889 que estaba a cargo del Ing. Carlos Fader, padre del reconocido pintor Fernando Fader-(Diario Los Andes 1921), para luego iniciar su explotación, producción del cemento puzolánico hacia mediados del siglo XX (Sironi 2016).

Los resultados de los trabajos de campo y gabinete permitieron establecer los procesos de ocupación para dichos sitios. A partir de los distintos hallazgos arqueológicos en los trabajos de campo se han realizado esquemas cronoestratigráficos correspondientes a lapsos históricos de MPS y MLA con diferentes escalas de tiempo, los que son producto de la relación estratigráfica y profundidades (promediadas y generalizadas para todas las excavaciones) de los emplazamientos mineros mencionados y que permiten establecer criterios de sincronía relativa (Tabla 1).

En MPS, las ocupaciones se atribuyen a tres lapsos temporales. La primera es del período republicano de fines del siglo XIX y primera década del XX. Esta fase está representada por materiales cerámicos (vidriadas tipo carrascal) y restos de vidrio de botellas de ginebra cuadradas, además de clavos y otros elementos metálicos como cartuchos de bala y piezas de herramientas manuales asociadas a labores mineras. La segunda fase corresponde a la primera mitad del siglo XX . En este caso se halló un zapato femenino, a lo que se suman abundantes restos de vidrio y metálicos. Menos abundantes, 
Tabla 1. Esquema cronoestratigráfico relativo a base de contextos y tipologías de materiales (vidrios, metales y lozas) definidos para MPS y MLA.

\begin{tabular}{lccc}
\hline Sitio arqueológico & Nivel estratigráfico & Profundidades $(\mathrm{cm})$ & Datación relativa \\
\hline \multirow{3}{*}{ Minas Paramillos Sur } & Nivel 1 & $0-20$ & $1950-1970$ \\
& Nivel 2 & $20-60$ & $1910-1950$ \\
& Nivel 3 & $60-100$ & $1870-1910$ \\
\hline \multirow{3}{*}{ Mina La Atala } & Nivel 1 & $0-35$ & $1940-1970$ \\
& Nivel 2 & $35-70$ & $1910-1940$ \\
& Nivel 3 & $70-120$ & $1890-1910$ \\
\hline
\end{tabular}

Fuente: Osvaldo Sironi (2015).

pero muy variables y fragmentadas son las lozas whiteware correspondientes a este período. En la tercera fase se incluyen elementos subactuales como fragmentos vítreos y metálicos (Sironi 2015, 2018).

En el caso de MLA, las ocupaciones iniciales se atribuyen a fines del siglo XIX (por lo menos desde 1890), representada por escasos restos de vidrio de botellas de vino jerez. La segunda fase corresponde a la primera mitad del siglo XX, evidenciada por abundantes restos de vidrio y elementos metálicos, escasas lozas whiteware y un zapato masculino. La tercera fase corresponde, al igual que MPS, a elementos subactuales como vidrios y metales (Sironi 2016, 2018).

\section{Distribución y circulación del espacio minero}

Es admisible mencionar que, usualmente, la aplicación de los análisis gamma y de circulación (índice de Blanton) son realizados sobre edificios individuales que presentan varias habitaciones y que son emplazadas en contextos urbanos. A causa de las particularidades y aislamiento que presentan las estructuras mineras aquí analizadas, hemos optado por tomar los conjuntos o recintos como una unidad, es decir, como una sola estructura, ya que partiendo desde las plataformas o aterrazados (espacios al aire libre, construidos con el objetivo de configurar la interacción social como así también la distribución de las distintas habitaciones), podremos observar las simetrías y asimetrías y la distribución de las lógicas sociales configuradas en los espacios mineros analizados del NO de Mendoza.

\section{Minas Paramillos Sur (MPS)}

De acuerdo con los análisis murarios realizados en MPS se determinaron dos tipos de edificación.
Los recintos denominados Estructuras III y IV (área habitacional-administrativa) presentan una construcción por superposición regular que alterna rocas en hiladas simples, con bases muy grandes a las que se superponen otras hiladas de rocas de tamaño menor. Se observó un trabajo de canteado y de medidas estandarizadas en las rocas que componen la caja muraria de dichos recintos. Los conjuntos arquitectónicos restantes responden a un patrón edilicio que presentan muros simples con hiladas de piedra, intercalando rocas de tamaños grandes y medianos. En ciertos casos, los muros se fabricaron por medio de rocas dispuestas en hiladas paralelas y el entremedio fue rellenado por barro y pedregullo -lo que sirvió de aglutinante- con un espesor de $90 \mathrm{~cm}$ aproximadamente (muros dobles con relleno). En los casos que los muros presentan solamente una hilada de rocas, el espesor de los mismos alcanza $70 \mathrm{~cm}$, aproximadamente. En ambos casos se comprobó que los muros estaban asentados en mortero de barro.

En el relevamiento detallado de los muros pudimos establecer diferencias constructivas que respondieron a diferentes fases o modificaciones y ampliaciones del complejo minero. Inicialmente se diferencian dos conjuntos arquitectónicos que se asientan en una plataforma nivelada y están vinculados, correspondiendo al núcleo central del sitio histórico-arqueológico analizado. Las Estructuras III y IV -recintos simples- están divididas por un patio interno que se fusiona en el aterrazado público que albergó una tinaja de agua de grandes dimensiones; y los dos conjuntos arquitectónicos (Estructuras V y VI) se encuentran separados por un camino que desemboca en el aterrazado público. Es decir, los recintos y conjuntos se diferencian en la construcción y distribución de las habitaciones, aunque, en una escala general, se construyeron manteniendo la 
idea de integrarlos a partir de la configuración de caminos, senderos y nivelados del terreno, los que se visualizan en el patio interno entre la Estructura III y IV, y en el aterrazado público con funciones de áreas de descarte (Sironi 2015).

Para profundizar una lectura de la arquitectura y la organización del espacio del emplazamiento minero MPS, confeccionamos planos y detalles de los edificios, los que permiten sustentar las hipótesis referidas al proceso de construcción y transformación del terreno para el emplazamiento de MPS. Utilizamos como punto de partida el relevamiento de planta, que es analizada a partir de modelos generados desde la arquitectura y las ciencias sociales que permiten observar la descomposición de la organización espacial expresada en gráficos y diagramas (Figuras 3 y 4 ).

La aproximación al edificio se advierte, en primer lugar, por la existencia de caminos y senderos que condicionan el sentido y dirección de la circulación. Existe una configuración del recorrido para todo el sitio que sigue un sentido principalmente lineal y secundariamente radial, es decir, hay una organización espacial que condiciona el movimiento desde un centro (aterrazado público) hacia las construcciones. Desde el acceso del exterior hacia el emplazamiento administrativo-habitacional de MPS era posible apreciar las Estructuras III y IV. De esta manera, cobraban relevancia tanto por su relación directa con el recorrido como por su mayor grado de exposición. Estas estructuras controlaban el acceso al lugar de extracción de minerales (socavones) y los sectores de habitaciones y cocina (Estructuras V y VI).

Los espacios destacados en el esquema general de circulación se encuentran: a) directamente relacionados a los accesos principales del lugar (Estructuras III y IV); o b) vinculados a la dirección y movimiento al situarse al final del recorrido (Estructuras V y VI). Predomina la aproximación frontal, esto es, que en cada estructura se conduce directamente a la entrada que se bifurcan en senderos de circulación del emplazamiento minero MPS. En cuanto al acceso de la mayoría de las estructuras hacia el espacio interior, se interrumpen por los cambios de nivel del suelo debido a la presencia de un escalón que obstaculizan la entrada.

Como se observa en la Figura 3 con su posterior descomposición en el mapa gamma (Figura 4), las Estructuras V y VI presentan el mayor grado de

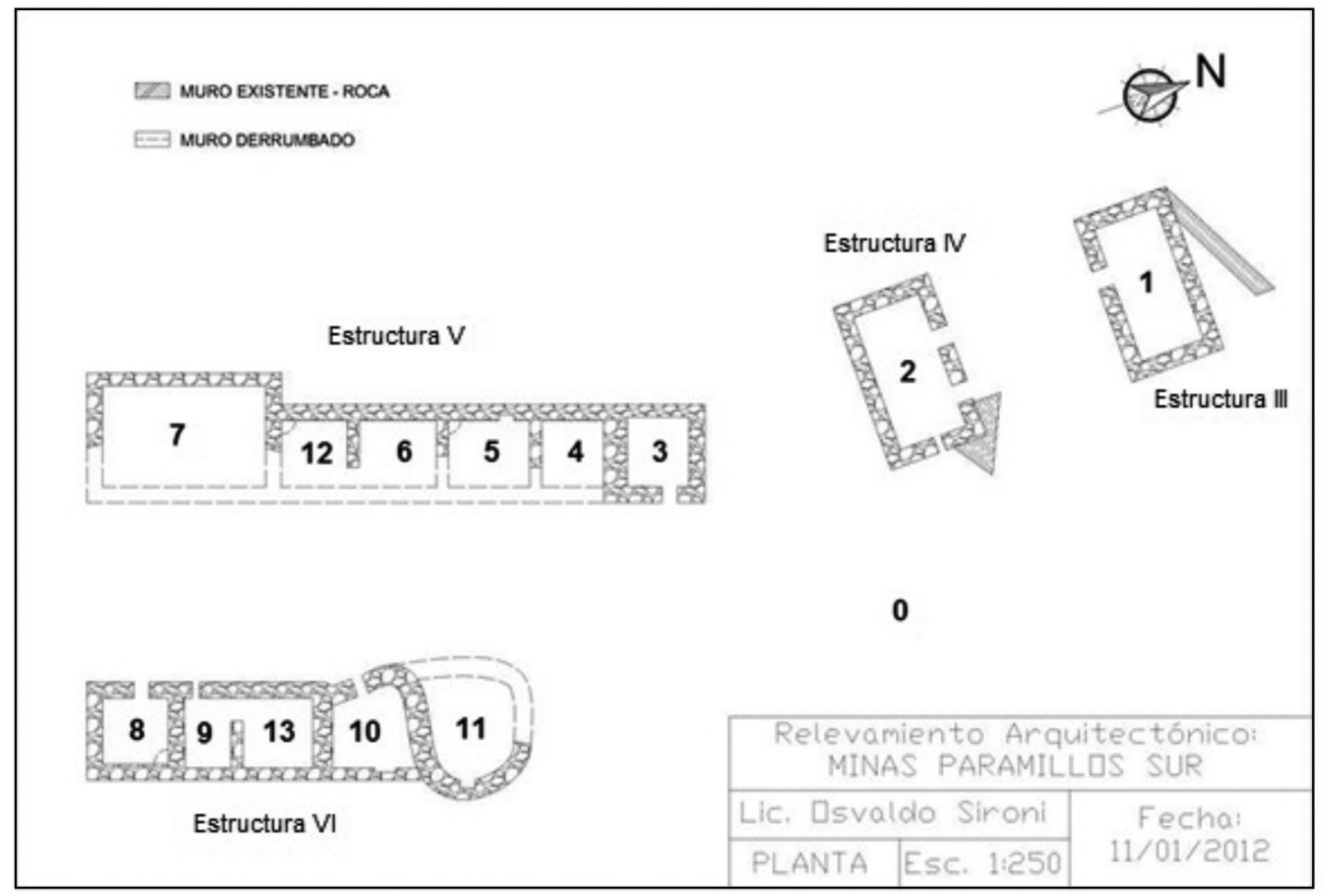

Figura 3. Planta de las estructuras domésticas relevadas en MPS. Los números indican los nodos considerados en cálculos de índices. Fuente: Osvaldo Sironi y Sofía Dutto (2012). 


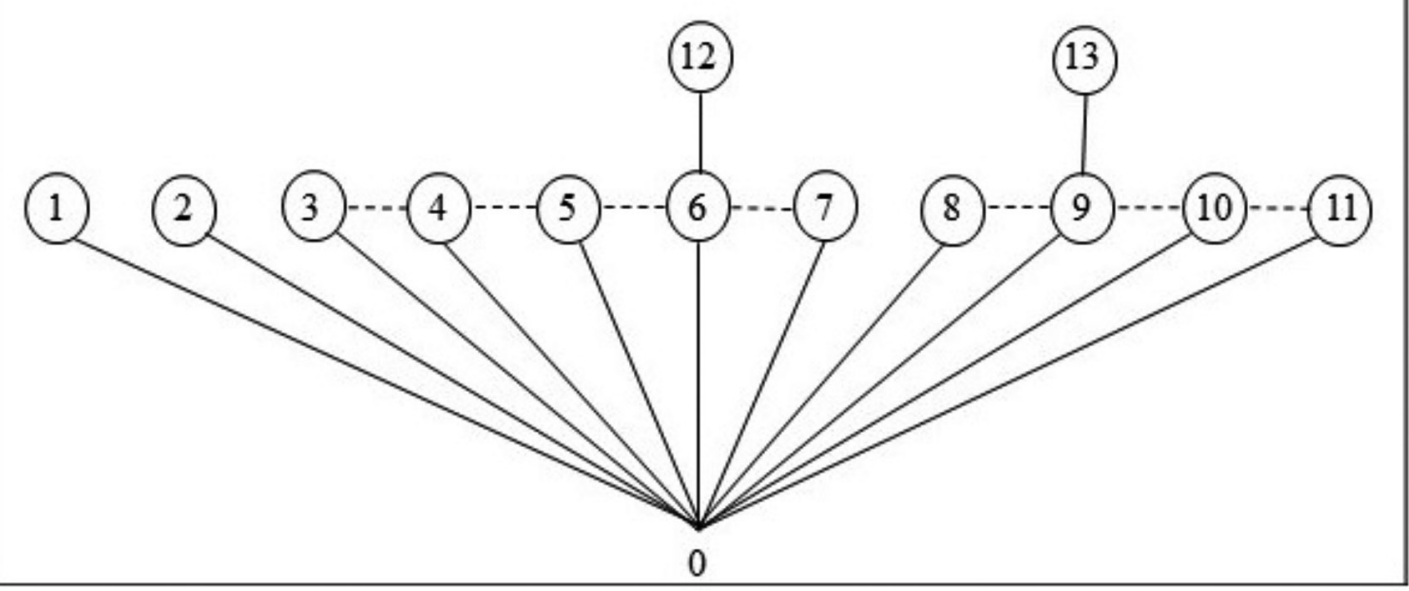

Figura 4. Gráfico derivado del análisis gamma acerca de recintos y conjuntos arquitectónicos administrativos-habitacionales de MPS. Fuente: Osvaldo Sironi (2015).

compartimentación del espacio expresado en el mayor índice de escala. En cuanto al índice de integración, este permite dar cuenta que las estructuras presentan el número mínimo posible, ya que cada cuarto tiene al menos una conexión. Sin embargo, solo dos habitaciones (nodos 12 y 13) revelan un grado de restricción y aislamiento de las restantes habitaciones (espacios bipermeables). En este conjunto arquitectónico se materializa una relación asimétrica y no distributiva entre los espacios, ya que su comunicación e integridad son muy bajas y los recorridos están obligados a ser transitados a partir de los aterrazados públicos.

$\mathrm{Si}$ bien las tendencias generales observadas en los índices de integración y de complejidad (según cantidad de conexiones por un lado y de accesibilidad de cada nodo con el exterior) poseen una caracterización propia de la modernidad (Zarankin 1999), al analizar estas situaciones se detectan ciertas particularidades que es necesario aclarar y podrían deberse a su situación en un emplazamiento productivo, diferente al de una construcción urbana.

El alto índice de escala (13 nodos) está mostrando un grado de compartimentación del espacio, dividido de tal manera que permita maximizar elementos de aislamiento como habitaciones. Esta estructura revela al mismo tiempo la necesidad del centro de generar un espacio celularizado como eje para su funcionamiento y control de los trabajadores. Por su parte, los índices de integración y complejidad evidencian que se trata de espacios no distributivos y con alto grado de aislamiento. Este tipo de estructuración es típico de instituciones disciplinares y autoritarias, donde existe un espacio de circulación controlado y regulado, como es el caso de la arquitectura "tipo cuartel" en emplazamientos mineros. Los diagramas a partir de la cuantificación de los nodos y conexiones de cada una de las estructuras arrojan un valor asimilable a los ambientes predominantes en la era del Capitalismo Industrial, a partir de los índices definidos por Zarankin (1999: 125).

La instalación del núcleo central (Estructuras III, IV, V y VI) parece responder a un diseño planificado, o al menos, a un ordenamiento espacial respetado en sucesivos agregados. Los arreglos lineales de habitaciones adosadas que comunican directamente a un espacio público podrían asimilarse al diseño "tipo cuartel" que caracteriza a las instalaciones mineras industriales europeas desde mediados del siglo XIX y luego replicado en distintos espacios mineros americanos (Sierra Álvarez 1985, Meléndez y Quesada 2012).

Por otra parte, el espacio de las unidades administrativas-habitacionales puede ser dividido en 2 ejes. Un sector inferior (que ocupa aproximadamente el $50 \%$ de la superficie total), que podemos denominar como el centro logístico-administrativo del emplazamiento (Estructuras III y IV) y otro sector superior (Estructuras V y VI), que aloja a los trabajadores mineros y donde se ubica la sala de cocina-comedor (nodo 11). De este modo, esta organización divide y clasifica a las personas dentro del emplazamiento, delimitando espacios de circulación y permanencia de los mineros. Asimismo, 
estas unidades administrativas-habitacionales absorben la esencia de las personas transformándolas en meros objetos en los que actúan los dispositivos del poder. Pensemos que la existencia social de cualquier persona está directamente relacionada con la posibilidad de interactuar con otros, de reconocer y ser reconocida.

En definitiva, en la instalación minera de MPS se observa una estructuración espacial con un núcleo de control de circulación, probablemente relacionados con las funciones y las interacciones, en ciertos ámbitos favorables a la homologación (patio de uso común, el que funciona como espacio de interacción social y a la vez de control a las habitaciones y el entorno circundante) y en otros a la diferenciación (habitantes de conjunto y no habitantes del mismo).

\section{Mina La Atala (MLA)}

La arquitectura del sitio es de piedras de procedencia local (Estructuras I, II, VI y X), adobe (Estructura III), ladrillos (Estructuras IV y X) y hormigón (Estructuras V, VII y X). Las piedras de procedencia local se encuentran ligadas con barro y pedregullo; los ladrillones de adobe han sido aglutinados con barro, fibras vegetales y pedregullo; y las restantes estructuras han sido ligadas con cemento y cal.

El núcleo doméstico-productivo-Complejo Aestá comprendido por tres estructuras (Estructuras I, II y III). En el sector oeste de este núcleo se hallan un horno-Estructura I- y un recinto cegado por los aluviones -Estructura II-. Hacia el este de las mismas se encuentra el conjunto arquitectónico-estructural de adobe, compuesta de cuatro habitaciones, y debido a las evidencias arqueológicas halladas confirmamos su funcionalidad doméstica -Estructura III-. Es importante destacar que frente a este núcleo doméstico-productivo, concretamente hacia el sur del mismo y frente al horno, se halla el Jirón B (socavón de esquistos bituminosos), el que permite un acceso directo al yacimiento mineral con una mínima inversión de trabajo, ya que el traslado del mineral hacia el horno de fundición se encuentra a escasos 16 metros, aproximadamente.

El Complejo B está comprendido por seis estructuras (Estructuras IV, V, VI, VII, VIII y IX), de estas, tres son rectangulares, dos circulares y una cuadrada. Este complejo arquitectónico responde a un patrón funcional vinculado exclusivamente al productivo, ya que se pueden observar un horno de ladrillo y dos bases rectangulares de hormigón enfrentadas a dos bases circulares que corresponderían a cañerías o silos. Respecto de la estructura cuadrada no hemos podido inferir su funcionalidad específica.

El complejo arquitectónico C también responde al patrón productivo, ya que está compuesto por una sola estructura (Estructura X), la que evidencia en su interior una plataforma rectangular de hormigón y metal de $1 \mathrm{~m}^{2}$. Dicha estructura posee una ubicación prioritaria en el paisaje respecto de los complejos A y B, ya que es el punto de concentración de los caminos. Esta ubicación nos permite inferir su funcionalidad de acopio para la distribución del producto obtenido en las explotaciones mineras.

En este caso, los análisis murarios permitieron establecer dos tipos de edificación:

1) Las Estructuras I, II, VI y X presentan una construcción de hilera simple, en donde se alternan rocas de distintos tamaños, con bases grandes, a las que se les superponen otras de menores dimensiones. Esta técnica constructiva se denomina opus incertum, en la que se define a partir de rocas desiguales en las que solo se cuida un poco su cara exterior y la mampostería suele estar compactada con mortero de barro o cal. Sin embargo esta generalidad encuentra una excepción en la edificación de la Estructura X, donde se observa un trabajo de combinación entre piedras canteadas adecuándose a la traba lograda, aparejos de ladrillos (columnas aglutinadas con cemento y cal) y aparejos de rocas locales (muros ligados con barro y pedregullo), y en el centro del recinto se visualiza una base de hormigón y metal. Las columnas de ladrillos fueron realizadas mediante la técnica aparejo a soga y tizón. Esta técnica se caracteriza por la disposición alternada de los bloques de ladrillos colocados de modo horizontal; unos por su lado más largo -soga- y otros por el más corto -tizón-. Es decir, el aparejo a soga es aquel en que vemos el canto del ladrillo, y las hiladas se superponen solapando a medio ladrillo la hilada anterior, para interrumpir la junta vertical; y el aparejo a tizón son todos los ladrillos que se colocan con la cara corta hacia afuera, también alternando juntas.

Las Estructuras IV y IX corresponden a recintos de ladrillos con funcionalidades productivas, como ser un horno y una especie de pileta para acumulación de agua. Las técnicas constructivas corresponden al aparejo a soga y tizón. 
2) La Estructura III -conjunto arquitectónico doméstico en donde se hicieron los análisis sintácticos- presenta medidas estandarizadas en los adobes, aglutinadas con barro, fibras vegetales y pedregullo. Estos se asientan en rocas grandes dispuestas en hiladas de superposición regular, que permiten una traba adecuada para la consolidación de los muros. Las técnicas constructivas aplicadas al levantamiento de estos paramentos de adobe son los denominados aparejos a tizón, aunque las columnas presentan la combinación de aparejo a soga y tizón. Detectamos diversas fases de acondicionamiento y enlucido de muros que responden a diferentes etapas de ocupación de dichas habitaciones.

En el relevamiento detallado de los muros pudimos establecer, entonces, diferencias constructivas, las que entendemos que pudieron responder a diferentes fases o modificaciones y ampliaciones del complejo minero. Inicialmente se diferencian los tipos de materiales utilizados para el levantamiento de los muros de los recintos, aunque estos últimos se encuentran en plataformas niveladas con empedrados, las que evidencian las transformaciones del terreno para la construcción del paisaje minero de MLA. Estas plataformas niveladas se encuentran protegidas mediante distintos empedrados que se distribuyen sobre la barranca del Arroyo Divisadero, los que protegían al emplazamiento minero de las escorrentías y aluviones. A pesar de esto, los habitantes sufrieron dichas escorrentías y aluviones, estas generaron inundaciones de las habitaciones domésticas en MLA, ya que las mismas se ubican en un corte del cerro. La expoliación de la vegetación que contribuía a frenar las avenidas de aluvión que bajaban de los cerros, aceleró los procesos de erosión del piedemonte, provocando riesgos en la población de dicho asiento minero.

Los recintos se encuentran interconectados por senderos o caminos. Es importante destacar que el complejo A podría representar el primer asentamiento para la explotación de los esquistos bituminosos y el complejo B y $\mathrm{C}$ responden a las características arquitectónicas para la producción del cemento puzolánico. De este modo, podemos afirmar que el conjunto arquitectónico A fue refuncionalizado para la adaptación a nuevos ritmos y tipos de producción, es decir, la obtención del esquisto bituminoso para autoabastecimiento de la puesta en marcha de la maquinaria para la producción del cemento puzolánico hacia mediados del siglo XX.

Por las características del relieve pedemontano donde se implanta el sitio se ha identificado una ruta de movimiento con dirección sudeste-noroeste, como se puede observar en el siguiente plano (Figura 5).

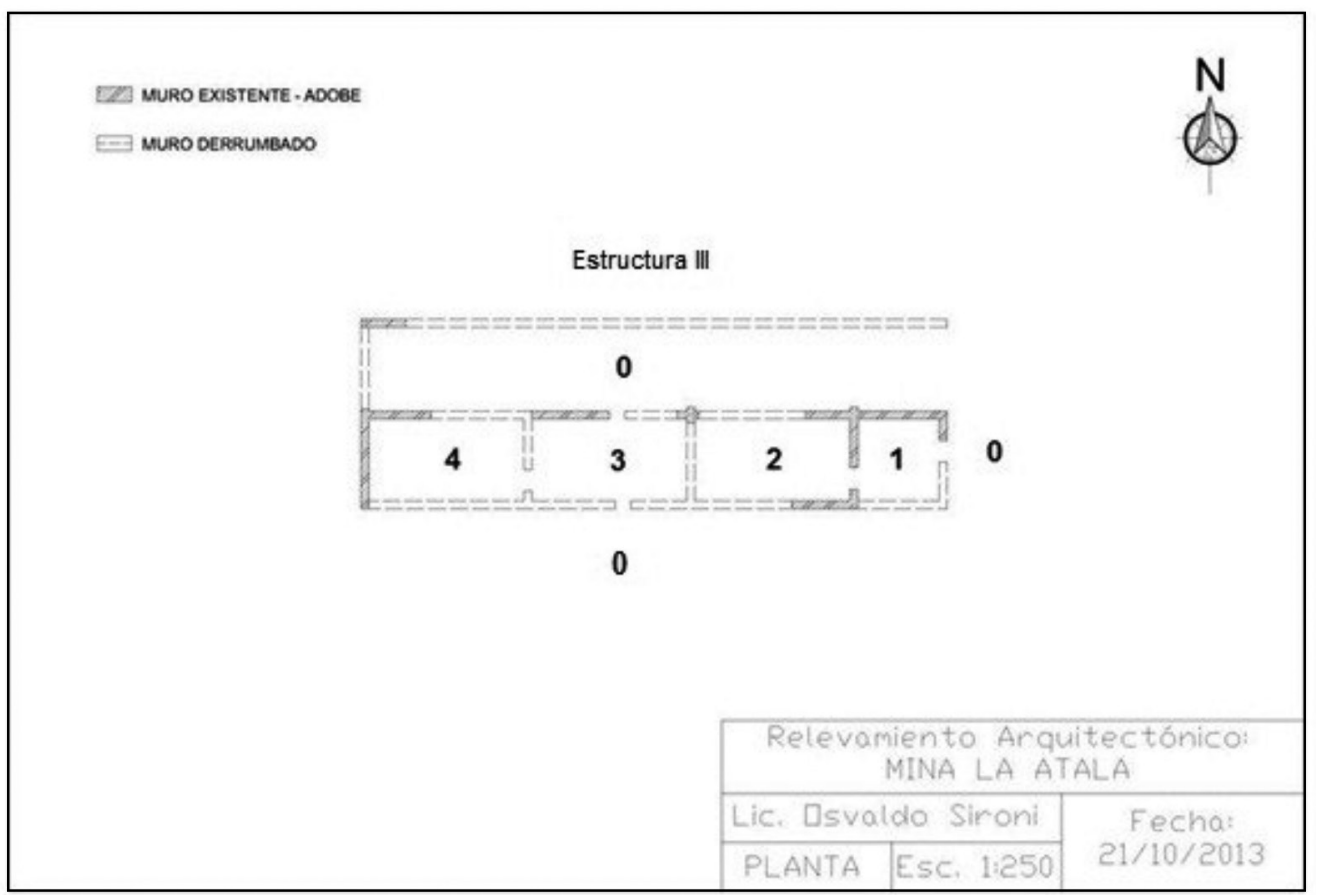

Figura 5. Planta de las Estructura III en MLA. Los números indican los nodos considerados en cálculos de índices. Fuente: Osvaldo Sironi y Sofía Dutto (2013). 
En el estudio de dependencia, la distribución y la visibilidad, se pudo observar que se trata de una construcción en la que el paso de un espacio a otro está únicamente controlado por el espacio inmediatamente contiguo. Según la sistematización de Hillier y Hanson (1984), esto implica que los espacios del área doméstica-habitacional tienen una relación asimétrica, pues para llegar a la habitación 2 es necesario pasar antes por la habitación 1 . La misma lógica se aplica en las habitaciones 3 y 4 (Figura 6).

En esa totalidad de habitaciones, el índice de integración arroja un valor asimilable a las casas predominantes en la era del Capitalismo Industrial (Zarankin, 1999: 125), dado por un índice de 1,75 (7 conexiones/4 nodos).

\section{Discusión}

Al comparar los índices de escala y compleji$\mathrm{dad}$, las tendencias entre ambos sitios presentan una clara diferencia, en tanto el índice de integridad es relativamente proporcional (Figura 7).

La comparación entre espacios (y entre la manera de percibirlos y la forma de acceder a ellos) es lo que posibilita establecer cómo está organizado el sistema habitacional del emplazamiento minero MLA, detectando cuáles son las relaciones entre los espacios y cómo son los modos de distribución, es decir, cuáles son las áreas más visibles (y por tanto sociales) y cuáles son las más reservadas. Este punto, aunque solo sea una aproximación inicial, es de gran relevancia ya que en los modos de distribución se están percibiendo en realidad modos de control e interacción social en el conjunto edificado.

Parte del acierto en las suposiciones que se hagan depende, sin embargo, de la fase de acercamiento inicial y la tipología de espacios resultantes. En términos generales, de acuerdo con el análisis gamma, los espacios más visibles, las habitaciones 1 y 3 permiten una vida pública (mayor interacción social), mientras que las habitaciones 2 y 4 son espacios segregados y controlados. Otros aspectos comparables entre los sitios son los siguientes:

- Tendencia al uso de materias primas locales para ambos emplazamientos. Asimismo, en MLA se utilizan materiales como adobe (en construcciones domésticas) y ladrillos y rocas (en construcciones productivas).

- Delimitación y organización funcional del espacio, según sectores de extracción, producción, acopio y habitacional. Las instalaciones domésticas-administrativas de MPS y MLA responden a un diseño planificado, el que pretende mantener a los trabajadores en su interior y separados de aquellas racionalidades productivas. El diseño tipo cuartel conformaba un dispositivo de disciplinamiento económico

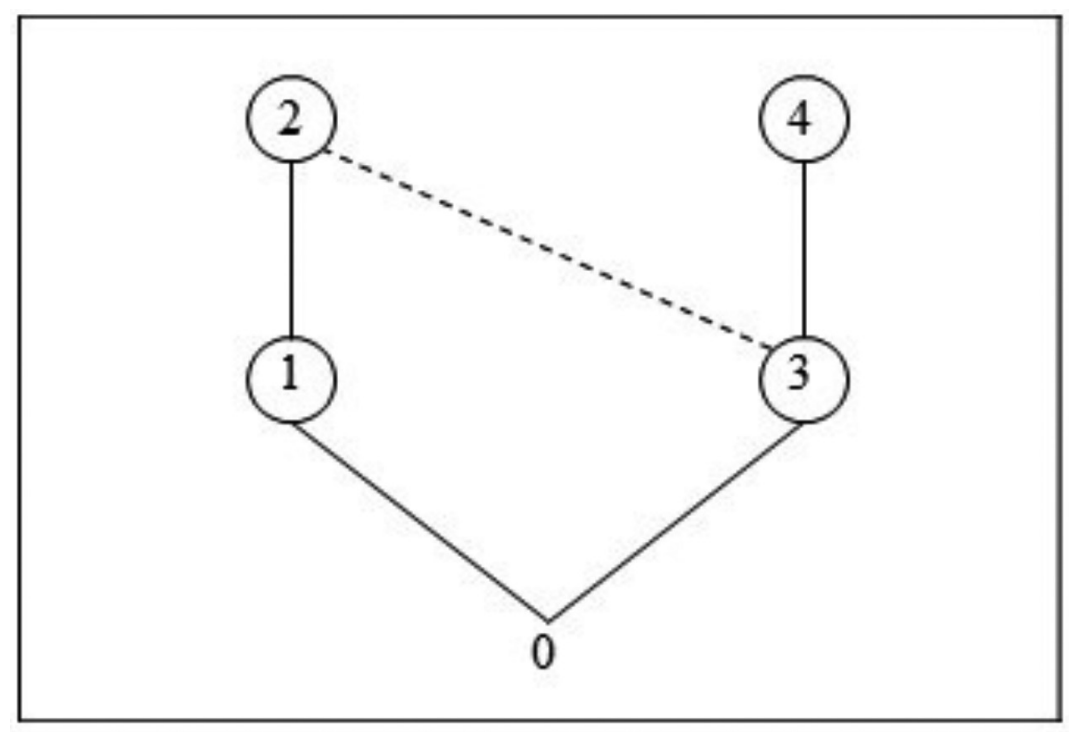

Figura 6. Gráfico derivado del análisis gamma sobre la Estructura III de MLA.

Fuente: Osvaldo Sironi (2015). 


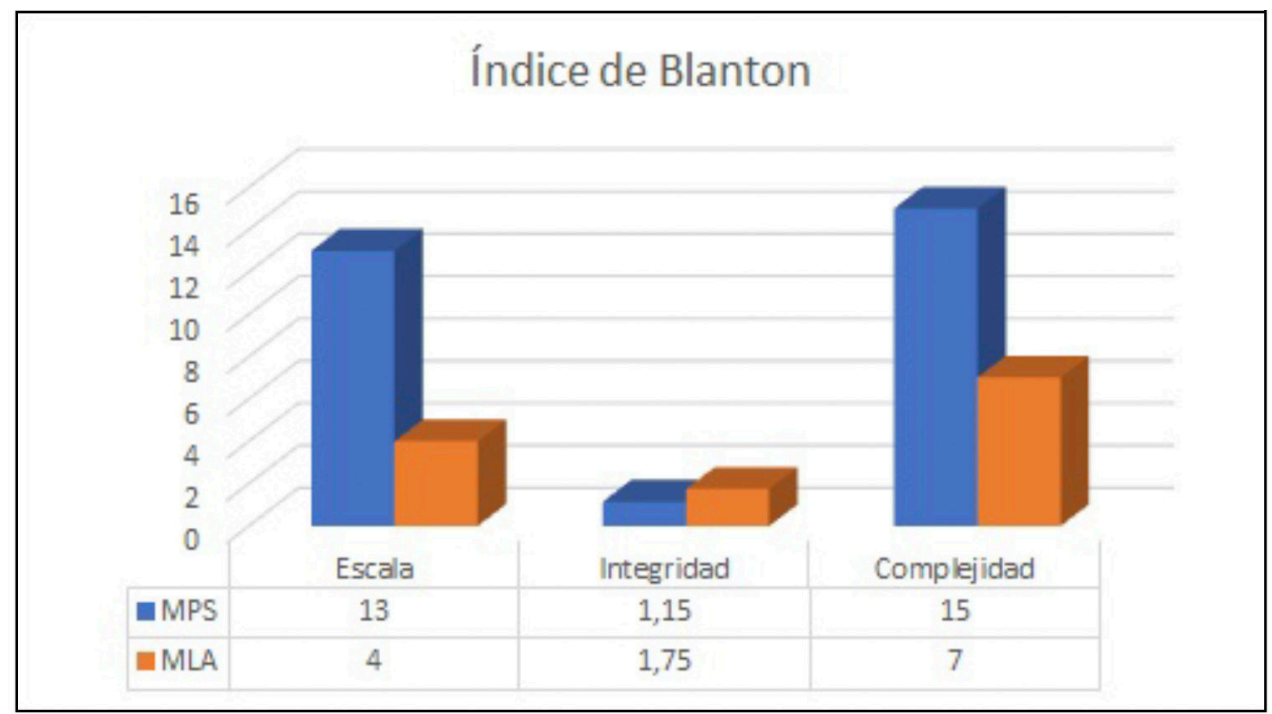

Figura 7. Comparación de Índices de Blanton entre las estructuras de MPS y MLA. Fuente: Osvaldo Sironi (2015).

y de fácil vigilancia, ya que su acceso inmediato a espacios públicos reducía al mínimo la privacidad.

- Evidencias de marginación laboral entre el proletariado industrial minero de MPS y MLA, en un contexto sociopolítico de disciplinamiento de mano de obra implementado durante el contexto histórico abordado.

\section{Consideraciones}

Con el objetivo de comprender la estructuración y lógica sociocultural del espacio y del paisaje minero precordillerano de Mendoza, realizamos los primeros estudios comparativos entre diferentes estructuras arquitectónicas, a partir de la aplicación de los análisis gamma (Hillier y Hanson 1984, Blanton 1994). Además del análisis morfológico y tipológico de las unidades domésticas-productivas, se comprendieron el proceso y técnicas de construcción involucradas, para luego analizar su composición y la distribución de los espacios. Ahora bien, el desarrollo y aplicación de estas categorías analíticas arquitectónicas al ámbito productivo extraurbano -en nuestro caso, la minería-, no solo contempló el punto de vista individual de cada una de las construcciones a las que fue aplicado, sino que fue puesta en relación con las demás. Cada construcción, sea emplazamiento, habitación o ambiente, fue entendido como un objeto con capacidad de relacionarse con el medio en el que está construido y con el resto de objetos que le rodean, es decir, teniendo en cuenta las diversas características de los grupos sociales a los que corresponden dichos espacios habitacionales.

No podemos afirmar taxativamente que los sitios analizados fueron habitados de modo permanente o estacional. Sin embargo, en el relevamiento de superficie se detectaron acumulaciones de residuos -restos de minerales, fragmentos de herramientas manuales mineras y restos de objetos de la vida cotidiana-asociadas a las estructuras. Por esta razón y por las características arquitectónicas postulamos que las mismas se relacionaron con actividades productivas y domésticas simultáneas realizadas durante tiempos extendidos de uso a lo largo del año. Un argumento a favor de esta hipótesis es que los conjuntos edilicios se encuentran organizados y en relación de control directo a los piques, socavones y galerías de las minas detectadas, como así también presentan una delimitación y organización funcional del espacio, según sectores de extracción, procesamiento y vida doméstica (aunque esta se solapa con áreas de descarte -basureros-). Los mismos se recorren por sendas interconectadas que los vinculan y que ubican a los edificios en puntos intermedios. Las características de los muros, su tamaño y técnicas de elaboración, si bien indican una fuerte inversión de trabajo, no habrían implicado el uso de tecnología y maquinaria moderna, 
presentando un patrón de edificación que tiende al uso de materias primas locales. Rocas indistintamente para construcciones domésticas y productivas en MPS y diferenciadas entre ladrillos, rocas y adobes según fueran espacios de producción o vivienda en MLA. Tendemos a pensar en una construcción en la que el trabajo está implicando sobre todo la fuerza muscular humana y a lo sumo la de algún animal (mulas o caballos), debido al difícil acceso y al tipo de técnicas constructivas aplicadas.

En el contexto de la producción minera capitalista, como es el caso de MPS y MLA, se define una tendencia a la reutilización y acondicionamiento de la arquitectura. Las secuencias de uso de estos espacios (intermitentes, continuas o estacionales), permiten estimar que el agua y su disponibilidad predecible en un contexto árido, gravitó como elemento decisivo en la selección y emplazamiento de los emprendimientos mineros y modos de organizarse espacialmente (más allá del mineral objeto de explotación que determinaba la necesaria cercanía), como es el caso de MPS, a diferencia de MLA que se enclava en terrenos surtidos con aguas permanentes. Sin embargo, esas tendencias a reutilizar y acondicionar varían debido a la disponibilidad de materiales constructivos y costos de obtención (rocas, argamasa, adobe, barro, ladrillos, cemento puzolánico), siendo claro que siempre se apuntó a bajar costos incluso manteniéndose muy por debajo de los umbrales de riesgo aceptables (se trata de zonas expuestas a sismos o aluviones).

De esta manera, más allá de la existencia de una articulación directa entre la configuración arquitectónica y espacial de MPS y MLA y las estrategias de control de los trabajadores, se constata que esta relación tiene un accionar concreto en distintos planos, permitiéndonos postular que en los emplazamientos mineros estudiados aquí, un componente esencial a estas estrategias la constituyó la manipulación de la cultura material y la configuración de los contextos laborales. En efecto, MPS y MLA se configuraban arquitectónica y espacialmente en función de los intereses económicos de las comunidades mineras, representadas por los dueños de los medios de producción y los que poseían los derechos de explotación de dichos minerales, en tanto se adaptó y adoptó un patrón de arquitectura "tipo cuartel", el que permitía el control de los accesos y caminos del lugar, aumentando el cerramiento y aislamiento de las habitaciones, segmentando los espacios y maximizando el uso de estos. Estas conclusiones demuestran que un riguroso análisis arquitectónico constituye una excelente vía de ingreso para comprender, en complementación con los estudios de cultura material citados, como se produjo y reprodujo la vida social en contextos del avance capitalista en las periferias.

\section{Agradecimientos}

A los miembros del Centro de Investigaciones Ruinas de San Francisco (Municipalidad de Mendoza). A la Universidad Nacional de Cuyo y CONICET por los apoyos institucionales y fondos. A la Dirección de la Reserva Natural Villavicencio, la Dirección de Recursos Naturales Renovables de la Provincia de Mendoza y la Dirección de Patrimonio. A Sofía Dutto y Pablo Cahiza por la digitalización de las plantas arquitectónicas y la elaboración de la cartografía. A los/as evaluadores/as por sus comentarios y/o sugerencias que permitieron mejorar sustancialmente el trabajo.

\section{Referencias Citadas}

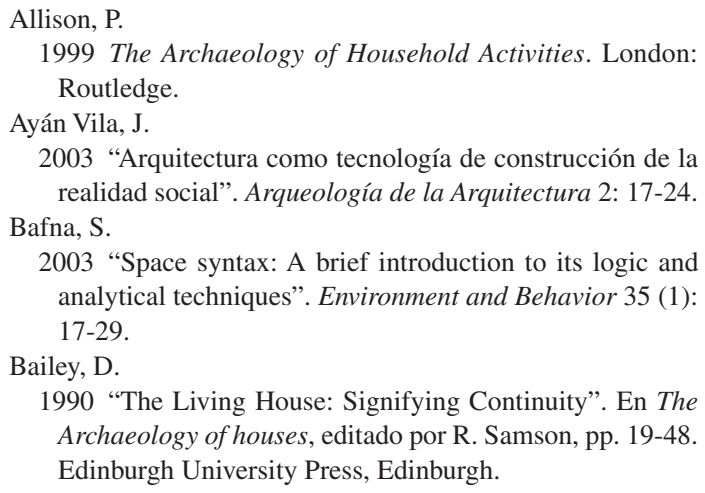

Baxter, R.

2002 "Industrial and domestic landscapes of a California oil field”. Historical Archaeology 36 (3): 18-27.

Blanton, R.

1994 Houses and Households: a comparative study. Plenum Press, New York.

Cabrera, A. y A. Willink

1980 Biogeografia de America Latina. Organizacion de los Estados Americanos, Washington DC.

Chiavazza, $\mathrm{H}$.

2010 "Arqueología de un emplazamiento rural: estancia San Pablo; Mendoza, Argentina (s. XVIII-XX)". Revista de Arqueología Histórica Argentina y Latinoamericana y Argentina 4: 135-168. 
Chiavazza, H. y C. Prieto Olavarría

2012 "Mineros en la puna de Mendoza: Arqueología del sitio Los Hornillos (Reserva Natural Villavicencio, Mendoza)". Vestigios. Revista Latino-Americana de Arqueología Histórica 6 (1): 115-153.

Ching, F.

2000 Arquitectura: Forma, espacio y orden. Editorial Gustavo Gili, Barcelona.

Chiotti, O.

1946 Estratigrafía y tectónica del Oeste de la ciudad de Mendoza y Las Heras. Tesis Doctoral, Facultad de Ciencias Exactas y Naturales, Universidad Nacional Córdoba. Inédita. Criado Boado, F.

1991 "Construcción social del espacio y reconstrucción arqueológica del paisaje". Boletín de Antropología Americana 24: 7-29.

Criado Boado, F. y Mañana Borrazás, P.

2003 "Arquitectura como materialización de un concepto: la espacialidad megalítica". Arqueología de la Arquitectura 2: $103-112$.

Cueto, A. O.

2003 "Minería y Sociedad. La minería y su incidencia social en Mendoza. Siglos XVI-XVIII". En Minería e Impacto en Mendoza, compilado por A. Cueto, pp. 57-80. Editorial de la Facultad de Filosofía y Letras de la Universidad Nacional de Cuyo, Mendoza.

Diario Los Andes.

1921 La minería. Número especial: Vistazo retrospectivo a la región de Cuyo al cerrar el año 1920: 97-112. Mendoza.

Eco, U.

1968 La estructura ausente. Introducción a la semiótica. Editorial LUMEN, Barcelona.

Fletcher, R.

1989 "The Messages of Material Behaviour: a Preliminary Discussion of Non-verbal Meaning". En The Meaning of the Things: Material Culture and Simbolic Expression, editado por I. Hodder, pp. 33-39. Unwin Hyman, London.

Foucault, M.

1976 Vigilar y Castigar. El Nacimiento de la Prisión. Siglo XXI editores, México.

Fuenzalida Bahamondes, N.

2011 "Cuartel Terranova, análisis de la configuración espacial en relación a las estrategias de represión y control de detenidos y torturados". La Zaranda de Ideas. Revista de Jóvenes Investigadores en Arqueología 7: 49-63.

Funari, P. y A. Zarankin.

2003 "A social archaeology of housing from a Latin American perspective: a case study". Journal of Social Archaeology 3 (1): $23-45$.

Grilli, D.

2006 "Paramillos de Uspallata (Mendoza, Argentina): su explotación a través de la arqueología y la historia”. En Patrimonio geológico y minero: su caracterización y puesta en valor, editado por I. Rábano y J. M. Mata-Perelló, pp. 79-90. Instituto Geológico y Minero de España, Madrid.

Harrington, H. y De Benedetti, J.

1941 "Investigación geológica en las sierras de Villavicencio y Mal País (Mendoza)”. Boletín de la Dirección Nacional de Geología y Minería 49: 1-56.

Hillier, B. y J. Hanson

1984 The Social Logic of Space. Cambridge University Press, Cambridge.
Hodder, I.

1994 "Architecture and meaning: The Example of Neolithic Houses and Tumbs". En Architecture and Order. Approaches to Social Space, editado por Parker Pearson, M. y C. Richard, pp. 73-86. London: Routledge.

Kent, S.

1990 Domestic Architecture and the use of Space: An interdisciplinary cross-cultural study. Cambridge University Press, Cambridge.

Lallemant, G.

1890 La minería en la provincia de Mendoza: El Paramillo de Uspallata. Imprenta Coni, Buenos Aires.

Mañana Borrazás, P.; Blanco Rotea, R. y Ayán Vila, X.

2002 Arqueotectura 1: Bases teórico-metodológicas para una arqueología de la arquitectura. Universidad de Santiago de Compostela, Santiago de Compostela.

Meléndez, S. y M. Quesada

2012 "Estrategias empresariales y tácticas campesinas en Mina Dal". Vestigios. Revista Latino-Americana de Arqueología Histórica 6 (1): 157-186.

Nastri, J.

1997-1998 "Patrones de asentamiento prehispánicos tardíos en el Sudoeste del Valle de Santa María (Noroeste argentino)". Relaciones de la Sociedad Argentina de Antropología XXI- XXIII: 247- 270.

Palmer, M. y Neaverson, P.

1998 "The scope of industrial archaeology". En Industrial Archaeology Principles and Practice, editado por M. Palmer y P. Neaverson, pp. 1-15. Routledge, London.

Reynoso, C.

2010 Análisis y diseño de la ciudad compleja. Perspectivas desde la antropología urbana. Editorial SB, Buenos Aires.

Rolón, G.

2013 La vivienda popular riojana del ámbito rural. Patrones arquitectónicos y contexto social en los valles durante el Período Republicano. Tesis doctoral presentada en la Facultad de Filosofia y Letras de la Universidad de Buenos Aires, Argentina.

Samson, R.

1990 The Social Archaeology of Houses. Edinburgh University Press, Edinburgh.

Sánchez, J.

1998 "La arqueología de la arquitectura. Aplicación de nuevos modelos de análisis a estructuras e la Alta Andalucía en Época Ibérica". Trabajos de Prehistoria 55 (2): 98-109.

Sierra Álvarez, J.

1985 "Política de vivienda y disciplinas paternalistas industriales en Asturias". ERIA 8: 61-71.

Sironi, O.

2013 "El impacto de los medios de producción minera en los Paramillos de Uspallata (Mendoza, Argentina): Un acercamiento histórico en la larga duración (siglos XVII-XIX)". Revista de Historia Americana y Argentina 48: 57-97.

2015 "Intervenciones arqueológicas en el sitio Minas Paramillos Sur (Reserva Natural Villavicencio, Mendoza)". En Arqueología y Etnohistoria del Centro Oeste argentino: Aportes desde las V Jornadas arqueológicas cuyanas, editado por J. R. Bárcena, pp.119-133. Zeta editores, Mendoza.

2018 “Arqueología Industrial en Mina 'La Atala' (Reserva Natural Divisadero Largo, Mendoza)". Revista de Arqueología Histórica Argentina y Latinoamericana 12: 835-861. 
2019 "Mining Ways of Life in the Southern Andes: Historical Anthropological Archaeology in Mendoza, Argentina". International Journal of Historical Archaeology 23 (1): 153-171.

Steadman, S.

1996 "Recent research in the Archeology of architecture: Beyond the Foundations". Journal of Archaeological Research, 4 (1): 51-93.

Wynveldt, F.

2005 "Análisis espacial de los conjuntos arquitectónicos de la Loma de los Antiguos de Azampay (Departamento de Belén, Catamarca)". En Azampay: presente y pasado de un pueblito catamarqueño, editado por M. C. Sempé, S. Salceda y M. Maffi, pp. 381-411. Ediciones Al Margen, La Plata.
Zarankin, A.

1999 "Casa Tomada; Sistema, Poder y Vivienda Familiar". En Sed Non Satiata. Teoría social en la Arqueología Latinoamericana Contemporánea, editado por F. Acuto y A. Zarankin, pp. 239-272. Ediciones del Tridente, Buenos Aires.

Zarankin, A y C. Niro

2009 "La materialización del sadismo: Arqueología de la arquitectura de los centros clandestinos de detención de la dictadura militar argentina (1976-1983)”. En Arqueología de la Represión y Resistencia en América Latina (19601980), compilado por P. Funari y A. Zarankin, pp. 159-182. Editorial Brujas, Córdoba.

\section{Nota}

1 Con el fin de ampliar la mirada teórico-metodológica aplicada en este tipo de análisis, realizados en contextos urbanos o rurales a escala nacional e internacional, sugerimos consultar las obras de Bailey (1990), Hodder (1994),
Allison (1999), Mañana Borrazás et al. (2002), Ayán Vila (2003), Criado Boado y Mañana Borrazás (2003), Reynoso (2010), Fuenzalida Bahamondes (2011) y Rolón (2013). 
